\title{
SÉANCE DU 11 AVRIL 1919
}

Présidence de M. BOIS, Vice-Président.

M. le Président, encore souffrant, s'est fait excuser de de ne pouvoir assister à la séance.

M. R. Mirande, secrétaire, donne lecture du procèsverbal de la séance précédente dont la rédaction est adoptée.

M. le Président annonce le décès de MM. Cogniaux et Janczewski. Ces deux éminents botanistes, qui ne faisaient pas partie de notre Société, lui ont témoigné leur intérêt par l'envoi de plusieurs de leurs ouvrages.

M. le Président annonce ensuite deux nouvelles présentations :

MM. Bonsin (Adelphe), rue Hubert-Latham, $\mathrm{n}^{\circ}$ 12, à Châteauneuf-en-Thymerais (Eure-et-Loir), présenté par MM. de Litardière et Cousturier.

Galinat (Maurice), étudiant, rue de Fleurus, $\mathrm{n}^{\circ} 3$, à

Paris, VI', présenté par MM. Lutż et F. Camus.

MM. Bonnin et Galinat sont proclamés, séance tenante, membres de la Société.

M. le Secrétaire général donne lecture du compte rendu de l'état financier pendant ces dernières années. 


\section{État des recettes et des dépenses de la Société du $1^{\text {er }}$ janvier 1915 au $1^{\text {er }}$ janvier 1918.}

Au 31 décembre 1914, l'actif de la Société se composait de:

Rentes nominatives $2.630 \mathrm{fr}$. ayant coùté. . . 75.03715

- au porteur $\frac{110 \mathrm{fr} .}{2.740 \mathrm{fr} .} \ldots \ldots . . .5 \frac{3.59710}{78.63425}$

Dépôt au Comptoir d'Escompte . . . . . . 1.110 65

Numéraire Trésorier. . . . . . . . . 3.80295

— $\quad$ Secrétaire général . . . . . $\quad 3.6962587 .20410$

Recettes en 1915 . . . . . . . . . . . . . . . $\frac{7.50615}{94.71025}$

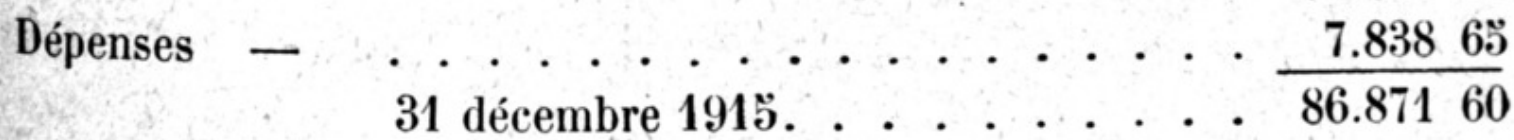

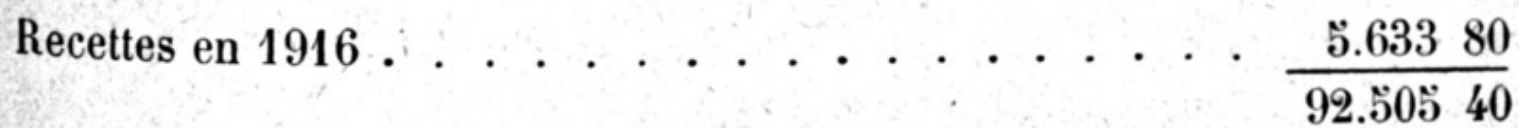

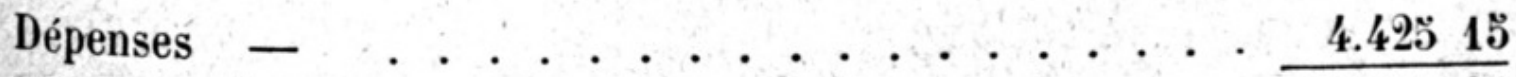

31 décembre 1916. . . . . . 88.08025

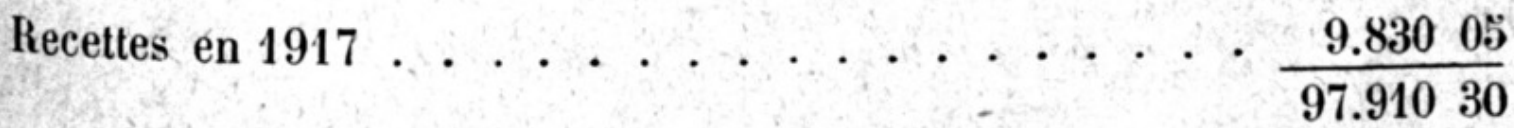

Dépenses _ . . . . . . . . . 6.66110

91.24920

Au 31 décembre 1917, l'actif de la Société se décompose comme suit :

Rentes nominatives $2.630 \mathrm{fr}$. ayant coùté. — 73.03715

$$
\begin{array}{ccc}
110 \mathrm{fr} . & \cdots & 3.59710 \\
\hline 2.740 \mathrm{fr} . & \cdots & 78.63425 \\
\text { min de fer d'Orléans. } & \cdots & 4.140
\end{array}
$$

Dépôt au Gomptoir d'Escompte. . . . . 1.15465

Numéraire Trésorier. . . . . . . . . 4.31460

- Secrétaire général. . . . 3.00370 


\section{Exercice 1915.}

\section{Recettés}

Cotisations annuelles.

2.82450

à vie.

150 . .

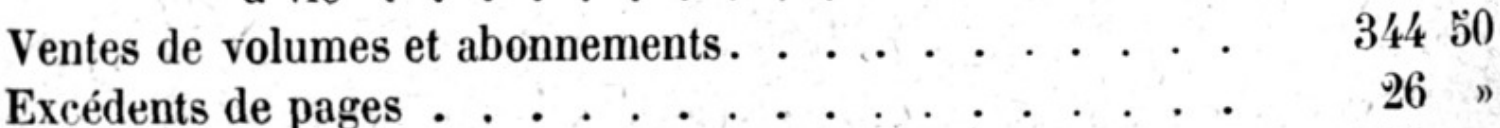

Subvention du Ministère de l'Instruction publique. . . . . 700 "

500 »

Rentes sur l'État et coupons Orléans . .

Intérêts du Comptoir d'Escompte

7.50615

Revue bibliographique

Frais de gravure

3075

Port du Bulletin.

Impressions diverses

Loyer et impositions

Chauffage et éclairage

10010

Prix de Coincy. .

Dépenses diverses.

Bibliothèque :

Dépenses extraordinaires

Honoraires du Rédacteur du Bulletiu . . . . . . . . 1.100 ”

Gages du garçon de bureau.

Exercice 1916.

Recettes.

Cotisations annuelles.

1.500 "

à vie.

$450-1$

Ventes volumes et abonnements .

9350

Subvention du Ministère de l'Instruction Publique

$700 \%$

Rentes sur l'État et coupons Orléans . 
DÉPENSES.

Impressions du Bulletin . . . . . . . . . . . . . . 1.46470

一 diverses. . . . . . . . . . 2710

Loyer . . . . . . . . . . . . . . . . . . . 1.35060

Dépenses diverses. . . . . . . . . . . . . . . 2775

Honoraires du Rédactetur du Bulletin . . . . . . . . 1.300 "

Gages du garçon de bureau. . . . . . . . . . 20ั "

Total ............ . . 4.42515

Exercice 1917.

ReCETTES.

Cotisations annuelles. . . . . . . . . . . . . . 1.920 ,

Ventes de volumes et abonnements. . . . . . . . 185"

Subvention du Ministère de l'Instruction publique . . . . 700 "

Rentes sur l'État et coupons. . . . . . . . . . . 2.88250

Intérêts du Comptoir d'Escompte . . . . . . . . . 25

Legs de M. Malinvaud . . . . . . . . . . . . . . . . $4.140 ”$

Total ............. $\frac{49805}{9.83005}$

DÉPENSES.

Impression du Bulletin. .............. . . . 2.954 ”

diverses................ . . 2930

Loyer et impositions. . . . . . . . . . . . . 2.01225

Dépenses diverses. . . . . . . . . . . . . . . . . . $\quad 8490$

Honoraires du Rédacteur du Bulletin. . . . . . . $1.200 "$

Gages du garçon de bureau. . . . . . . . . . 330 "

Bibliothèque . . . . . . . . . . . . .

Total ............ . . 6.661 10

Notre Trésorier, M. Philippe de Vilmorin, a été mobilisé dès la première année des hostilités et est décédé au cours de l'année 1917. Notre confrère, M. Hibon, membre du Conseil, a bien voulu accepter provisoirement de gérer les finances de la Société. Nous ne saurions trop témoigner notre reconnaissance à notre confrère pour avoir consenti à accepter et pour avoir rempli d'une façon aussi satisfai- 
sante ces lourdes et délicates fonctions pendant une période de temps aussi difficile.

M. Bois donne lecture de la Notice suivante :

\section{Notice sur M. Maurice Lévêque de Vilmorin;}

PAR M. D. BOIS.

La Société botanique de France a fait une perte très regrettable en la personne de M. Auguste-Louis-Maurice Lévêque de Vilmorin.

Elle le comptait parmi ses membres depuis 1878 et l'appela à présider ses travaux en 1911.

Son frère, M. Henry Lévêque de Vilmorin, mort en 1899, avait été, lui aussi, président de notre Société et son neveu, M. Philippe Lévêque de Vilmorin, décédé en 1917, en fut trésorier.

I. Maurice de Vilmorin naquit à Verrières-le-Buisson (Seine-et-Oise), le 26 février 1849 ; il est mort le 21 avril 1918, au château des Barres (Loiret), au voisinage immédiat des admirables collections dendrologiques, au développement desquelles il consacra la plus grande partie de sa vie.

Continuateur zélé de l'œuvre des Vilmorin, il tenait dans l'Agriculture et l'Horticulture une très grande place, en raison de ses connaissances spéciales, de la droiture de son caractère, de sa bonté, de son affabilité, qu'une réserve dùe à une certaine timidité pouvait masquer à ceux qui l'dbordaient pour la première fois, mais qui ne tardait pas à s'affirmer ensuite.

Dans le demi-siècle qui vient de s'écouler, il a pris une grande part à l'accroissement de nos richesses dans le domaine de l'Arboriculture forestière et d'ornement par l'introduction et la propagation d'espèces et de variétés rares ou nouvelles. Il était en contact permanent avec les botanistes de la France et de l'étranger, avec les voyageurs-naturalistes, les missionnaires, auxquels nos jardins sont si redevables; aussi avait-il sa place marquée dans une assoriation comme la nòtre, où les études de science pure se poursuivent parallèlement à celles des applications, à la satisfaction de lous, dans un but d'intérêt général.

C'est dans une propriété familiale, le domaine des Barres, près de Nogent-sur-Vernisson (Loiret), que M. Maurice de Vilmorin entreprit ses premières études dendrologiques en revisant les plantations d'arbres 


\section{$2 \mathrm{BHL}$ Biodiversity Heritage Library}

1919. "Séance Du 11 Avril 1919." Bulletin de la Société botanique de France 66, 136-140. https://doi.org/10.1080/00378941.1919.10836091.

View This Item Online: https://www.biodiversitylibrary.org/item/8686

DOI: https://doi.org/10.1080/00378941.1919.10836091

Permalink: https://www.biodiversitylibrary.org/partpdf/161135

\section{Holding Institution}

Missouri Botanical Garden, Peter H. Raven Library

\section{Sponsored by}

Missouri Botanical Garden

\section{Copyright \& Reuse}

Copyright Status: Public domain. The BHL considers that this work is no longer under copyright protection.

This document was created from content at the Biodiversity Heritage Library, the world's largest open access digital library for biodiversity literature and archives. Visit BHL at https://www.biodiversitylibrary.org. 